\title{
Diagnostic Accuracy in Venous Thrombosis
}

\author{
J. M. LAMBIE,* M.B., B.S. ; R. G. MAHAFFY,† F.R.C.S.ED., F.F.R. ; D. C. BARBER, $\ddagger$ M.SC., B.A. \\ A. M. KARMODY,* CH.M., F.R.C.S. ; M. M. SCOTT, $§$ M.B., CH.B. ; N. A. MATHESON, $\|$ CH.M., F.R.C.S.
}

\begin{abstract}
Cummary: A group of 111 surgical patients at high risk $\checkmark$ of venous thrombosis were studied after operation by independent clinical assessment and with ${ }^{125}$ I-fibrinogen to detect venous thrombosis. Almost half of the patients developed venous thrombosis. Of these, twothirds were not suspected clinically despite careful scrutiny. In the patients in whom a clinical diagnosis of venous thrombosis was made this diagnosis was falsely positive in a quarter. More than half of all thrombotic episodes were detectable on the day after operation.

The prevalence of venous thrombosis, together with the difficulty in diagnosing it, strongly supports the argument that a reduction in the incidence of pulmonary embolism must depend on widespread adoption of effective prophylaxis, especially in the large number of patients at high risk of venous thrombosis. Prophylactic trials must be objectively assessed, and it is in this field that the ${ }^{125 I-}$ fibrinogen technique probably has the most to offer.
\end{abstract}

\section{Introduction}

In the United States pulmonary embolism kills 47,000 patients each year (Coon and Willis, 1959). In Britain about 2,500 patients are known to die annually from pulmonary embolism (General Register Office, 1969); five times as many episodes were diagnosed in Oxford in 1961 as in 1952 (Morrell et al., 1963). The prevention of pulmonary embolism depends, if not on prophylaxis, at least on the early detection and treatment of venous thrombosis. The diagnosis of venous thrombosis is almost always clinical and is based on well-accepted symptoms and signs. The lack of clinical evidence of venous thrombosis in $50 \%$ of patients dying of pulmonary embolism (Sevitt and Gallagher, 1959), however, casts doubt on the usefulness of these signs. Despite this and other supporting evidence accruing from work with precise diagnostic techniques, currently a clinical diagnosis of venous thrombosis gives the only warning of the possibility of embolism in almost all the patients at risk. The failure of this warning in half of those who develop pulmonary embolism is sufficiently disquieting; but, in addition, clinical assessment has been the sole means of evaluation in most published studies in which prophylactic regimens against venous thrombosis have been compared. Most clinicians believe in frequent examination of the legs in patients confined to bed and particularly after operation. How far is this diagnostic faith justified? Apart from the value of precise information on the limitations of clinical diagnosis, we must be aware of these limitations if our approach to preventing pulmonary embolism is to be constructively reoriented.

In the present study the accuracy of clinical diagnosis of venous thrombosis was evaluated in 111 surgical patients who were studied with a precise diagnostic technique, ${ }^{125}$ I-fibrinogen being used.

\section{Methods}

We studied 111 patients thought to be at high risk of developing postoperative venous thrombosis. The group com-

* Registrar in Surgery.

+ Consultant Radiologist.

Terminable Lecturer in Medical Physics.

Senior House-officer in Surgery.

Consultant Surgeon.

University of Aberdeen and Royal Infirmary, Aberdeen. prised obese gynaecological patients aged over 45 years undergoing hysterectomy or pelvic floor repair, general surgical patients aged over 40 undergoing major abdominal surgery, and elderly patients having internal fixation of fractures of the femoral neck.

Clinical assessment was carried out in each patient by a single independent observer unaware of the result of radioisotope scanning. Two of us were responsible for this part of the work, each examining about half the total number of patients. The clinician assessed the patients on at least six of the first 10 postoperative days and recorded his findings on a form. The diagnosis of venous thrombosis was allowed to rest on the discretion of the clinician after repeated examination; no predetermined criteria were used.

\section{I-tagged Fibrinogen}

The incorporation of ${ }^{125}$ I-tagged fibrinogen into the fibrin matrix of an evolving thrombus allows the resulting area of increased radioactivity to be detected by scanning the legs with a collimated scintillation counter. Flanc et al. (1968) and Negus et al. (1968), with differences only in detail, have shown this technique to be accurate and reliable. ${ }^{125}$ I-tagged fibrinogen was prepared by the jet iodination method (McFarlane, 1958) the fibrinogen source being either fibrinogen (Kabi) or fibrinogen prepared from a single blood sample from a healthy donor by the triple precipitation method (McFarlane, 1963). Recently all fibrinogen has been prepared from a single blood sample. The ${ }^{125} \mathrm{I}$ was obtained, in a concentrated carrier-free solution specially prepared for protein iodination, from the Radio Chemical Centre, Amersham. Each patient was given $180 \mathrm{mg}$. of potassium iodide orally on the eve of operation, and this daily dosage was continued for two weeks after operation. Immediately after the operation $50 \mu \mathrm{Ci}$ of ${ }^{125}$ I-fibrinogen was injected intravenously, and the patient's legs were scanned on each of the first 8 to 10 days after operation. The scanning technique and the criteria used for the diagnosis were those described by Negus et al. (1968).

Ascending phlebography was performed in 46 patients with or without venous thrombosis (not all included in the 111 patients under discussion) to confirm the accuracy of the fibrinogen technique in our hands. Sixty-two phlebograms were obtained in 46 patients (both legs being examined in 16). In 18 legs the scanning indicated a clear venous system, and this was confirmed in 16 with phlebography. In two legs with a negative scan the phlebogram indicated the presence of thrombus in the deep veins. The age of the thrombus could not be determined, and both these patients had had previous episodes of superficial phlebitis. The thrombus shown by phlebography may therefore have been long-standing.

Of 44 legs with positive radioiodine scans, 40 were confirmed with phlebography, the correlation being consistent in both site and extent in each individual thrombus. One patient in whom phlebography was negative had obvious superficial phlebitis to account for the positive scan. In another patient, the finding of high counts but negative phlebography was explained by skin bruising. The two remaining patients showed high counts over one thigh. Phlebograms showing the superficial femoral vein were negative, but as there was no apparent cause for spuriously high counts it was thought possible that these patients had thrombus in the profunda femoris vein. Intraosseous phlebography to confirm or refute this possibility would not have been justified. 
When scanning was negative the findings were in agreement with those using phlebography in $89 \%$ of legs, and when the scanning was positive they were in agreement in $91 \%$ of legs. This degree of correlation is similar to that found in other studies (Flanc et al., 1968; Negus et al., 1968) and indicates that ${ }^{125}$ I-fibrinogen scanning is a reliable method for the examination of the clinical course, distribution, and frequency of venous thrombosis.

\section{Results}

The results are summarized in the Table. A firm clinical diagnosis of deep vein thrombosis was made in 25 of the 111 patients. In 18 of these 25 the clinical findings were supported by a positive scan and in 7 the clinical diagnosis was falsely positive. In 49 of the 111 patients ( $44 \%$ ), however, venous thrombosis was diagnosed on scanning. These 49 patients showed evidence of thrombosis in 61 legs, 12 patients having bilateral thrombosis. Of the 61 affected legs a clinical diagnosis was made in only 19 (18 patients, one having clinically declared bilateral thrombosis). Thus, despite close clinical scrutiny, $69 \%$ of thrombi were not detected.

Summary of Results in 111 High-Risk Patients

\begin{tabular}{|c|c|c|c|}
\hline & No. of Patients & & gs Involved \\
\hline $\begin{array}{l}\text { Positive clinical diagnosis ... } \\
\text { Confirmed with 1251-fibrinogen } \\
\text { Clinical false-positive diagnosis } \\
\text { Positive 125I-fibrinogen diagnosis .. } \\
\text { Clinical false-negative diagnosis .. }\end{array}$ & $\begin{aligned} 25 & (23 \%) \\
18 & (72 \%) \\
7 & (28 \%) \\
49 & (44 \%) \\
31 & (63 \%)\end{aligned}$ & $\begin{array}{r}26 \\
19 \\
7 \\
61 \\
42\end{array}$ & $\begin{array}{l}\text { (1 bilateral) } \\
(27 \%) \\
(12 \text { bilateral }) \\
(69 \%)\end{array}$ \\
\hline
\end{tabular}

Further analysis shows that in the 12 patients with bilateral thrombosis only one was correctly diagnosed, six had clinical signs in one leg only and the other five were clinically unsuspected of having venous thrombosis. Of the 61 legs affected 46 showed thrombi confined to the deep veins of the calf, and only 13 of these had clinical signs. Two patients had widespread thrombosis from groin to ankle. In one the diagnosis was clear clinically, but the other showed equivocal clinical signs; the thrombosis was clinically diagnosed on the eighth postoperative day, whereas the scan had been positive from the first postoperative day. Of the 61 thrombi 34 were initially suspected from the scan on the first day after operation, eight on the second day, and thereafter in decreasing frequency as the time from the operation increased.

Analysis of the relative accuracy of different clinical symptoms and signs in the diagnosis of deep vein thrombosis is of limited value, but serves to stress their inaccuracy. Symptoms were unhelpful: six patients complained of calf pain with no evidence of deep vein thrombosis on scanning, and only 3 of the 49 patients who developed thrombosis complained of calf pain. One patient had haemoptysis on the second postoperative day, but the subsequent findings and clinical course suggested infection rather than infarction. Chest pain was not found in this group of patients nor were any other subjective symptoms. Calf tenderness was present in 6 out of 61 legs with thrombosis and in 5 out of 161 without thrombosis. Homan's sign was positive in 14 out of 61 legs with thrombosis and in 2 out of 161 legs without thrombosis. Fever was noted in 14 out of 49 patients with thrombosis and in 30 out of 62 patients without thrombosis. Ankle oedema was noted in 18 out of 61 limbs with thrombosis and in 3 out of 161 limbs without thrombosis.

Changes in the circumference of the limb measured at standard points on the calf and the ankle indicate that the group of patients with thrombosis showed an increase in calf circumference significantly greater than that found in the patients without thrombosis. There was no definite difference in the ankle measurements. Only those patients with an increase in calf circumference of $3 \mathrm{~cm}$. or greater showed $100 \%$ correlation with the scan; this was noted in only 6 of the 61 legs with thrombosis. In 23 legs there was a measured increase of $1-3 \mathrm{~cm}$. at the calf-14 had deep vein thrombosis and 9 did not. In addition, 41 limbs affected with deep vein thrombosis showed an increase of less than $1 . \mathrm{cm}$. in circumference; 22 of them had no recorded increase. Increase in local skin temperature (as detected by the clinician's hand) and increased prominence of the surface veins were recorded in two and four patients respectively out of 49 with thrombosis. Lowenberg's sign (calf pressure test) was abandoned after two months as it was time-consuming and without obvious value.

\section{Discussion}

Of this group of high-risk patients almost half developed thigh and leg venous thrombosis. Two-thirds of these were never suspected clinically despite careful assessment. In those in whom a clinical diagnosis of thigh and leg venous thrombosis was made this diagnosis was falsely positive in a quarter. Thus even close and repeated clinical evaluation is highly unreliable, and little store should be set by it. It is better to be conscious of the large number of patients who have thigh and leg venous thrombosis despite the apparent normality of their legs. Indeed, the true incidence of venous thrombosis in patients such as ours may be even higher than we have found, since about nalf of the present patients were receiving prophylaxis with either oral warfarin or intravenous dextran 70 . In addition, it is jossible that some thrombi arising de novo in the external and common iliac veins were overlooked, since the scan obtained from this area is difficult to assess.

At present the prevention of pulmonary embolism depends largely on the early clinical diagnosis and treatment of deep vein thrombosis. On this basis it is clear that most patients with thigh and leg venous thrombosis and at risk of pulmonary embolism are untreated. Some also receive unnecessary treatment. It seems most unlikely that this problem can be resolved by the widespread adoption of a sophisticated diagnostic technique such as ${ }^{125}$ I-fibrinogen as was recently suggested (Kakkar et al., 1969). The technique is time consuming, and we cannot foresee its routine application. Furthermore, the treatment of established venous thrombosis is at present disappointing.

The results of the present investigation strongly support the argument that a reduction in the incidence of pulmonary embolism after operation must depend on vigorous and widespread adoption of effective prophylaxis against venous thrombosis, particularly in patients at high risk. The frequency with which thigh and leg venous thrombosis is detectable soon after operation indicates that prophylactic measures during operation are likely to be important, and, finally, it is in the objective evaluation of possible prophylactic measures that the ${ }^{125} \mathrm{I}$-fibrinogen technique is likely to be of most value.

We thank the consultant gynaecologists, orthopaedic surgeons, general surgeons, and the other medical and nursing staff both for permission to study patients under their care and for their cooperation.

We are indebted to Professor J. R. Mallard for advice and for providing facilities and equipment in the department of medical physics.

\section{REFERENCES}

Coon, W. W., and Willis, P. W. (1959). American foumal of Cardiology,

Flanc, C., Kakkar, V. V., and Clarke, M. B. (1968). British fournal of Surgery, 55, 742

General Register Office (1969). Statistical Review of England and Wales for the year 1967, Part 1, Tables Medical. London, H.M.S.O.

Kakkar, V. V., Howe, C. T., Flanc, C., and Clarke, M. B. (1969). Lancet, 2, 230.

McFarlane, A. S. (1958). Nature, 182. 53.

McFarlane, A. S. (1963). Fournal of Clinical Investigation, 42, 346.

Morrell, M. T., Truelove, S. C., and Barr, A. (1963). British Medical fournal, 2, 830

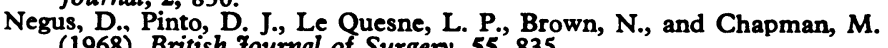
(1968). British fournal of Surgery, 55, 835.

Sevitt, S., and Gallagher, N. G. (1959). Lancet, 2, 981. 